\title{
Prediction of grain size, thickness and absorbance of nanocrystalline tin oxide thin film by Taguchi robust design
}

\begin{abstract}
Transparent conductive films of tin oxide were deposited on glass substrates under various deposition conditions. Taguchi analysis was used to model the dependence of the grain size, thickness and absorbance of nanocrystalline tin oxide on the process parameters namely $\mathrm{pH}$ value, concentration, time of deposition and bath temperature. The effect of the mentioned process parameters on the grain size, thickness and absorbance of deposited layer during the deposition of nanocrystalline was investigated using X-ray diffraction (XRD) technique, atomic force microscopy (AFM) and UV-Visible spectroscopy. Comparison between the model predictions and the experimental observations predicted a remarkable agreement between them. The predictions of the model and sensitivity analysis showed that among the effective process parameters, deposition time and concentration were the main parameters having significant effect on crystalline size. Bath temperature had the most significant effect on absorbance and deposition time had a dominant effect on thickness.
\end{abstract}

Keyword: $\mathrm{SnO} 2$ nanocrystalline; Grain size; Thickness; Absorbance; Taguchi robust design. 